\title{
Approche expérimentale par IRM et modélisation de la sédimentation et de la consolidation de la vase
}

\author{
Damien Pham Van Bang ${ }^{1}$, Emmanuel Lefrançois ${ }^{2}$, \\ Philippe Sergent ${ }^{3}$, François Bertrand ${ }^{4}$.
}

${ }^{1}$ Ingénieur-Chercheur, Centre d'Etudes Techniques Maritimes et Fluviales (CETMEF), Equipe Commune de Recherche LNHECETMEF, EDF/R\&D/LNHE, 6 quai Watier, BP 49, Bat K, 78401

Chatou Cedex.

${ }^{2}$ Maître de conférence, Laboratoire Commun d'Hydraulique

Numérique (LHN), Département de Génie des Systèmes Mécaniques, Laboratoire Roberval, UMR 6066 CNRS-UTC-CETMEF, BP 20529, 60200 Compiègne.

${ }^{3}$ Directeur Scientifique, Centre d'Etudes Techniques Maritimes et Fluviales (CETMEF), 2 Bd Gambetta, BP 60XXX, 60YYY Compiègne.

${ }^{4}$ Technicien CNRS, Laboratoire des Matériaux et des Structures du Génie Civil (LMSGC, Institut Navier, UMR 113 CNRS-LCPC-ENPC), Cité Descartes, 2 Allée Képler, 77420 Champs sur Marne.

\section{Résumé}

La sédimentation et la consolidation de suspensions, colloïdale et non-colloïdale, sont étudiées à partir d'un prototype vertical Spectromètre - Imageur par Résonance Magnétique (IRM). Le modèle mathématique de Gibson et une modélisation numérique 1D basée sur la méthode aux éléments finis (MEF) sont présentées. Une discrétisation 'Lax-Wendroff' en temps avec une technique de capture de choc (par une correction du flux, FCT) est utilisée pour décrire la propagation des fronts de concentration. Enfin, des comparaisons expériencesimulation sont proposées et discutées dans les cas de la suspension non colloïdale et colloïdale.

\section{$\underline{\text { Abstract }}$}

Settling and consolidation of colloidal and non-colloidal suspensions are investigated from a nuclear Magnetic Resonance Imaging (MRI) vertical prototype. The mathematical model of Gibson with a 1D numerical modeling based on the Finite Element Method (FEM) are presented. A time 'Lax Wendroff' discretization with a shock capturing technique (Flux Corrected Transport, FCT) is used for the propagation of concentration fronts. Finally comparisons between experiment and simulation are proposed and discussed for the case of noncolloidal and colloidal suspension. 


\section{Mots clés}

IRM, sédimentation, consolidation, éléments finis, Lax-Wendroff, FCT.

\section{$\underline{\text { 1. Introduction }}$}

Le phénomène de sédimentation est important à considérer dans les nombreuses applications (minières, chimiques, traitements de l'eau...) utilisant des suspensions de particules solides. Ce phénomène est particulièrement important pour la maintenance de certains aménagements ou ouvrages (chenaux de navigation, installations hydroélectriques...) mais aussi pour certaines études d'impact écologique (pollution, turbidité...).

Même si ce phénomène est depuis longtemps mis à profit par l'homme (par les anciens Egyptiens pour l'or, Bürger and Wendland [4]), il reste toutefois complexe à décrire. En effet, la sédimentation de petites particules n'a pas de solution mathématique exacte (Blanc and Guyon [3]) : les forces visqueuses entre les particules et le fluide environnant sont de très longues portées si bien qu'il est en pratique impossible de calculer les propriétés telles que la viscosité ou la vitesse de chute. En outre, la sédimentation des particules fines (colloïdales) est encore plus complexe à décrire à cause des phénomènes de floculation.

A une échelle macroscopique, la théorie de Kynch (1952) fondée sur la propagation des ondes cinétiques est largement utilisée. Elle est cependant incapable de décrire le processus de consolidation qui est non négligeable pour les suspensions argileuses (colloïdales). La théorie de Gibson (1967) la généralise en incluant la théorie de la consolidation des sols de Terzaghi (1923). L'équation de Gibson a été validée par des résultats expérimentaux issus de colonnes de sédimentation instrumentées d'émetteur-récepteur de rayons X (Bartholomeeusen et al [2]) ou de rayon $\gamma$ (Toorman et al [11]).

L'utilisation de l'IRM est en plein essor dans les applications nécessitant une technique rapide et non intrusive de la mesure de teneur en eau (Callaghan [5]). Son utilisation en rhéologie des fluides complexes (Raynaud et al [9], Rodts et al [10]) a déjà révélé l'apport de cette technique pour la vélocimétrie et les mesures de concentration. Dans cette étude, nous présentons brièvement la technique IRM pour enregistrer la sédimentation et la consolidation. Ensuite, la théorie de Gibson (sédimentation de Kynch et consolidation de Terzaghi) et le développement d'une méthode numérique pour la propagation des fronts de concentration sont détaillés. Enfin des comparaisons simulation-expérience sont discutées.

\section{Approche expérimentale par IRM}

\section{1. Matériaux et méthodes}

La sédimentation-consolidation de deux suspensions, l'une colloïdale (particules solides en interaction physico-chimique) et l'autre non colloïdale, est étudiée. La première est une suspension de kaolin (mélange poly-disperse de particules aplaties de dimension maximale 0.4-2 $\mu \mathrm{m}$, d'épaisseur 0.06-0.4 $\mu \mathrm{m}$ et de masse volumique $2.6 \mathrm{gcm}^{-3}$ ) dans de l'eau. La seconde est une suspension modèle (billes 
sphériques mono-disperse en polystyrène de diamètre $0.29 \pm 0.03 \mathrm{~mm}$ et de masse volumique $1.05 \mathrm{gcm}^{-3}$ ) dans de l'huile de silicone Rhodorsil (viscosité $20 \mathrm{mPa} . \mathrm{s}$ et densité $\left.0.95 \mathrm{gcm}^{-3}\right)$.
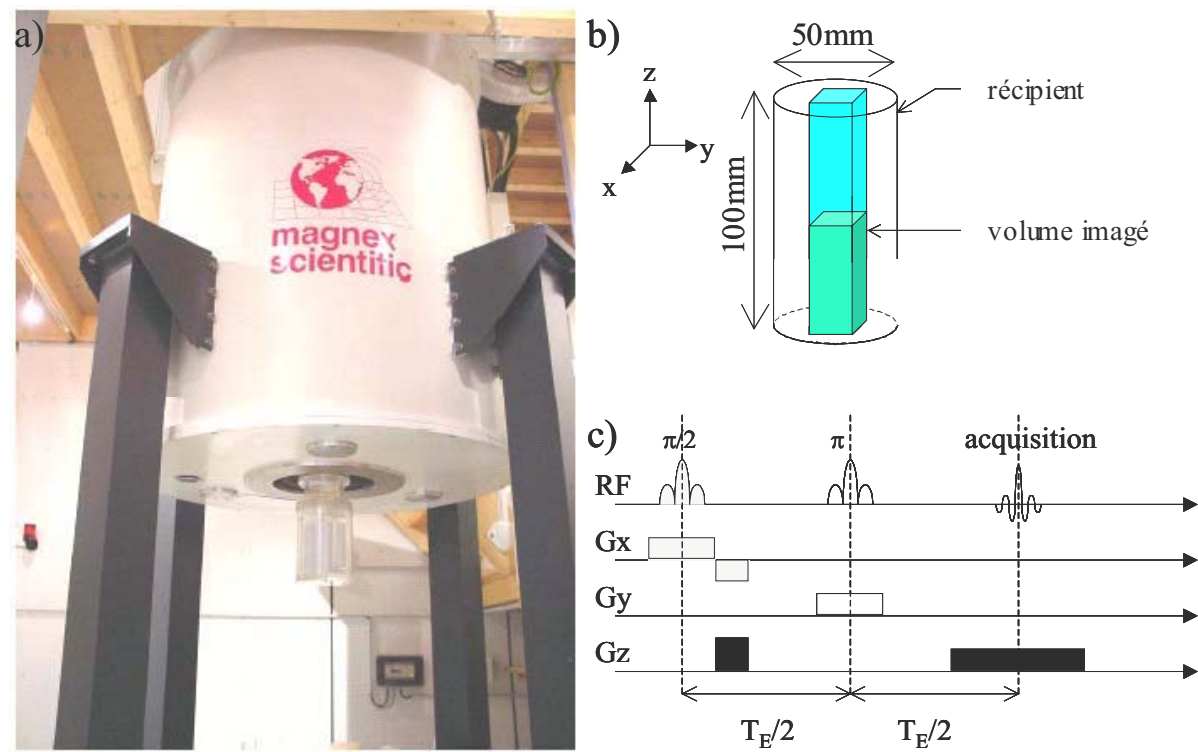

Figure 1: Principe de mesure du Proton IRM: a) Bruker 24/80 DBX spectrometer (LMSGC, Champs sur Marne, France); b) colonne cylindrique et volume imagé; c) séquence RMN inspiré de la méthode de vélocimétrie dans une poutre (Raynaud et al [9], Rodts et al [10]), éléments en gris (ou blanc) pour la sélection de la poutre dans la direction $\mathrm{x}$ (ou y respectivement) et élément en noir pour le 'gradient de lecture' vertical.

Les deux matériaux sont préparés dans un récipient cylindrique (diamètre 50 $\mathrm{mm}$; hauteur $100 \mathrm{~mm}$ ) pour une fraction volumique solide initiale $\left(\phi_{0}\right)$ égale à 0.18 pour la kaolinite et 0.48 pour la suspension modèle. Un malaxage (20 minutes à 300 tours/min) est appliquée pour obtenir une concentration initiale uniforme selon l'axe z, puis le récipient est couvert avant d'être placé dans l'IRM. Ce dernier effectue des mesures automatiques du profil de concentration le long de la verticale.

Les expériences sont réalisées au LMSGC (Champs sur Marne, France) sur un Bruker 24/80 DBX MRI (Figure 1a) opérant à $0.5 \mathrm{~T}(20 \mathrm{MHz}$ fréquence du proton) avec un aimant supraconducteur à axe vertical $(40 \mathrm{~cm})$ (Oxford, UK). La séquence RMN utilisée est basée sur une technique traditionnelle 'spin echo spin wrap' (Callaghan [5]) pour donner les mesures (profil vertical) de la densité de protons $\mathrm{H}^{+}$. Afin d'éviter les effets des parois, la séquence inclut deux excitations RF pour sélectionner un volume (Figure $1 \mathrm{~b}$ et 1c, section carrée de $20 \times 20 \mathrm{~mm}$ pour la kaolinite et $25 \times 25 \mathrm{~mm}$ pour la suspension modèle) et restreindre les mesures à l'intérieur de ce volume. La séquence $\mathrm{RMN}$ a un temps d'écho, $\mathrm{T}_{\mathrm{E}}$, de $7 \mathrm{~ms}$ pour la kaolinite $(6.9 \mathrm{~ms}$ la suspension modèle) : elle est répétée 8 (16) fois toutes les $5 \mathrm{~s}$ ( $3 \mathrm{~s}$ respectivement) pour obtenir un profil de concentration.

Donc, un simple profil d'intensité, $\mathrm{I}_{\text {susp }}(\mathrm{z})$, résulte d'une moyenne sur $40 \mathrm{~s}$ (48 s pour la suspension modèle) et donne une information sur la densité du fluide le long de la verticale. Une calibration à partir d'un profil de référence $\mathrm{I}_{\text {liq }}(\mathrm{z})$ obtenu 
sur le fluide pur permet alors une estimation de la fraction volumique solide à la profondeur z, Eq. (1):

$$
\phi(\mathrm{z})=\frac{\mathrm{I}_{\mathrm{liq}}(\mathrm{z})-\mathrm{I}_{\text {susp }}(\mathrm{z})}{\mathrm{I}_{\mathrm{liq}}(\mathrm{z})}
$$

Rigoureusement, les profils $I_{\text {susp }}(z)$ souffrent toujours d'un biais à cause du processus de relaxation de RMN qui se produit durant la séquence. De tels biais sont négligés dans la suite.

\section{2. Résultats expérimentaux}

a)

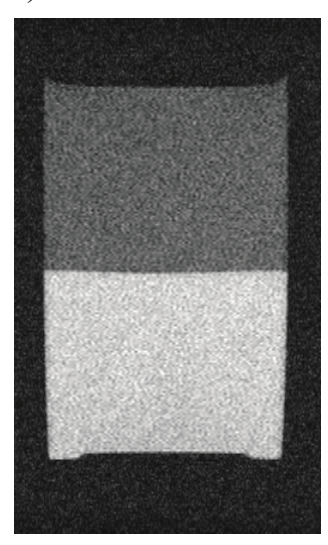

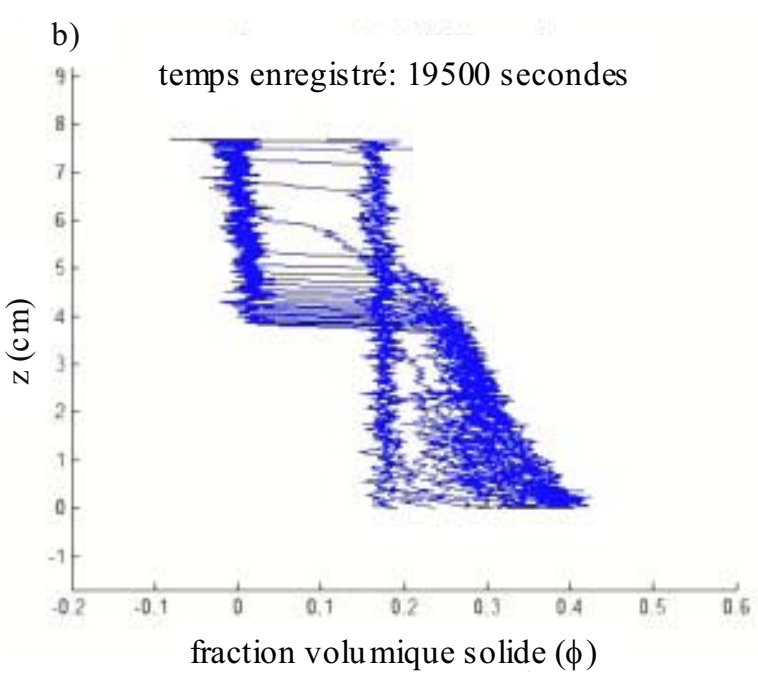

Figure 2: Résultats expérimentaux IRM sur la suspension de kaolin au temps 19500s : a) Image enregistrée (256x256 pixels); b) superposition des profils verticaux de concentration en utilisant Eq. (1).

A titre d'exemple, la figure 2 présente les résultats (image et profils de concentration obtenus par la technique IRM) jusqu'au temps $19500 \mathrm{~s}$ pour la suspension de kaolin. L'évolution des profils met en évidence les phénomènes de sédimentation et de consolidation. Cette évolution est en accord avec les résultats sur les sédiments fins obtenus par les techniques traditionnelles utilisant les rayons X ou $\gamma$ (Bartholomeeusen et al [2], Bürger and Wendland [4], Toorman et al [11]). Cette première observation valide ainsi qualitativement les mesures de profils de concentration obtenus par IRM.

D'autre part, considérant la durée (40 s pour une répétition de 8 échos) pour l'acquisition d'un profil de concentration, il est évident que la technique IRM possède une résolution temporelle nettement supérieure à celles des méthodes traditionnelles. Enfin, la résolution spatiale de la technique IRM est également supérieure aux techniques à rayon $\gamma$ ou $\mathrm{X}$ puisqu'un fin gradient de lecture est utilisé. Par conséquent, la technique IRM est particulièrement adaptée pour l'enregistrement et la capture de la propagation des fronts de concentration.

\section{Modélisations mathématique et numérique}

\section{1. Equation de sédimentation et de consolidation sous poids propre}


L'hypothèse d'un dépôt en deux régimes à priori simultanés (le premier correspond à la sédimentation de Kynch, le second à la consolidation de Terzaghi) amène plusieurs auteurs (Bartholomeeusen et al [2], Toorman et al [11]) à considérer l'équation de Gibson (Gibson et al [8]), Eq. (2).

$\frac{\partial \mathrm{e}}{\partial \mathrm{t}}+\left(\frac{\gamma_{\mathrm{s}}}{\gamma_{\mathrm{w}}}-1\right) \frac{\partial}{\partial \mathrm{e}}\left(\frac{\mathrm{K}}{1+\mathrm{e}}\right) \frac{\partial \mathrm{e}}{\partial \zeta}+\frac{\partial}{\partial \zeta}\left(\frac{\mathrm{K}}{\gamma_{\mathrm{w}}(1+\mathrm{e})} \cdot \frac{\partial \sigma^{\prime}}{\partial \mathrm{e}} \cdot \frac{\partial \mathrm{e}}{\partial \zeta}\right)=0$

où e est l'indice des vides, $\gamma_{\mathrm{s}}\left(\gamma_{\mathrm{w}}\right)$ le poids volumique de la phase solide (fluide respectivement), $\mathrm{K}$ est le coefficient de perméabilité (en $\mathrm{ms}^{-1}$ ), $\zeta$ est la coordonnée matérielle (lagrangienne) définie comme le volume solide par unité d'aire et mesuré dans la direction opposée à la gravité, et $\sigma^{\prime}$ est la contrainte effective.

Cette équation est dérivée des équations de conservation de la masse et du mouvement des deux phases, solide et liquide avec l'hypothèse d'un écoulement de Darcy à travers le sol et du principe de contrainte effective de Terzaghi. Elle exprime la sédimentation par le deuxième terme (terme de convection) et la consolidation de la mécanique des sols par le dernier terme (terme de diffusion).

Une généralisation 3D de cette équation avec la prise en compte d'un forçage hydrodynamique (par le courant ou la houle) et d'effets de diffusion (d'origine Brownienne et/ou turbulente) a été proposée en Eq. (3) (Toorman [9]) en terme de fraction volumique solide $(\phi)$ et en coordonnées eulériennes:

$\frac{\partial \phi}{\partial \mathrm{t}}+\frac{\partial}{\partial \mathrm{x}_{\mathrm{i}}}\left[\left(\mathrm{u}_{\mathrm{i}}-\delta_{\mathrm{i} 3} \mathrm{~K} \frac{\gamma_{\mathrm{s}}-\gamma_{\mathrm{w}}}{\gamma_{\mathrm{w}}} \phi\right) \phi\right]-\frac{\partial}{\partial \mathrm{x}_{\mathrm{i}}}\left[\left(\mathrm{D}_{\mathrm{s}}+\mathrm{D}_{\mathrm{s}}^{\mathrm{t}}\right) \frac{\partial \phi}{\partial \mathrm{x}_{\mathrm{i}}}+\delta_{\mathrm{i} 3} \frac{\mathrm{K} \phi}{\gamma_{\mathrm{w}}} \frac{\partial \sigma^{\prime}}{\partial \phi} \frac{\partial \phi}{\partial \mathrm{x}_{3}}\right]=0$

où $u_{i}$ est la composante $i$ de la vitesse de convection, $D_{s}\left(D_{s}^{t}\right)$ le coefficient de diffusion Brownienne (turbulente), et $\delta_{\mathrm{ij}}$ le symbole de Kronecker.

Deux lois constitutives sont finalement requises dans Eq. (2) et (3) pour exprimer la perméabilité et la contrainte effective en fonction de la fraction volumique solide: une variété d'expressions est proposée pour un même matériau (Bartholomeeussen et al [2] pour une revue).

A cette difficulté se rajoute le fait que la vitesse de chute de la suspension est ici reliée à la perméabilité ce qui est inadapté dans le cas des suspensions diluées (Toorman et al [9]). Finalement, comme rappelé par Gibson lui-même ([8]), la phase de consolidation secondaire est négligée si bien que les effets du temps (fluage et/ou le vieillissement) sont ignorés ; or leurs effets sont importants pour les suspensions argileuses.

\section{2. Code aux éléments finis avec la technique de correction de flux}

Le problème 1D à conditions initiale et aux limites est écrit par les équations (4) à (7), en considérant l'équation (4) comme une simplification de (3).

$$
\begin{aligned}
& \frac{\partial \phi}{\partial \mathrm{t}}+\frac{\partial}{\partial \mathrm{z}}[\mathrm{V}(\phi) \phi]-\frac{\partial}{\partial \mathrm{z}}\left[\mathrm{D}(\phi) \frac{\partial \phi}{\partial \mathrm{z}}\right]=0 \\
& \phi(\mathrm{z}, 0)=\phi_{0} \quad \forall \mathrm{z} \\
& \left.\phi(\mathrm{z}, \mathrm{t})\right|_{z=\mathrm{L}}=0 \quad \forall \mathrm{t} \\
& \left.\frac{\partial \phi(\mathrm{z}, \mathrm{t})}{\partial \mathrm{z}}\right|_{z=0}=0 \quad \forall \mathrm{t}
\end{aligned}
$$

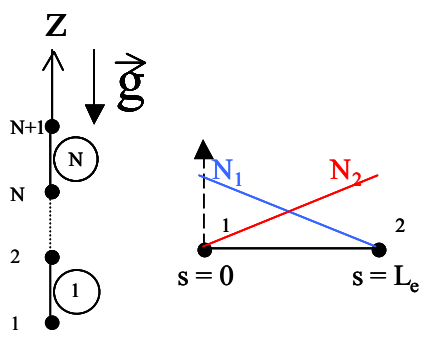


Figure 3: Eléments et noeuds pour le cas 1D.

Par application de la méthode aux éléments finis (Dhatt et al [6]), l'Eq. (8) est obtenue en considérant des éléments linéaires à deux nœuds (Figure 3). La discrétisation temporelle de (8) est basée sur un schéma de 'Lax-Wendroff' (LW, du second ordre en temps). Pour assurer la positivité du schéma, une technique de capture de choc est utilisée (Georghiou et al [5]). Elle est basée sur un algorithme FCT, initialement développé par Boris et Book, avec la procédure de limitation du flux de Zalesak:

$[\mathrm{M}]\left\{\Delta \phi^{\mathrm{n}}\right\}=\left\{\mathrm{R}^{\mathrm{n}}\right\}=-\Delta \mathrm{t} .([\mathrm{C}]+[\mathrm{K}])\left\{\phi^{\mathrm{n}}\right\}$

Le bon compromis entre la solution sans oscillation (schéma upwind) et la précision du schéma LW, particulièrement au voisinage d'un fort gradient voire d'une discontinuité en $\phi$, est obtenue par la construction d'un flux convectif net (point par point) comme une moyenne pondérée par un coefficient $\alpha_{i}^{\mathrm{n}}$ dans (9), d'une solution d'ordre faible (low) et d'une solution d'ordre élevée (high, ici la solution LW). Dans notre cas, la première est un schéma d'ordre un et la seconde est la solution de Lax Wendroff (LW) qui est d'ordre deux:

$\phi_{i}^{n+1}=\phi_{i}^{n}+\Delta \phi_{i}^{L n}+\alpha_{i}^{n} \cdot\left(\Delta \phi_{i}^{H n}-\Delta \phi_{i}^{L n}\right)=\phi_{i}^{n}+\left(1-\alpha_{i}^{n}\right) \cdot \Delta \phi_{i}^{L n}+\alpha_{i}^{n} \cdot \Delta \phi_{i}^{H n}$

où $\phi_{i}^{\mathrm{n}}$ est la valeur de la concentration, $\Delta \phi_{i}^{\mathrm{Hn}}\left(\operatorname{or} \Delta \phi_{i}^{\mathrm{Ln}}\right)$ la contribution de l'élément d'ordre élevé (high) ou faible (low), et $\alpha_{i}^{\text {n }}$ le coefficient de pondération variant entre 0 et 1 (indice pour le nœud $i$ et l'exposant pour l'instant $\mathrm{t}=\mathrm{n} \Delta \mathrm{t}$ ).

\section{Simulations et discussions}

\section{1. Comparaisons simulation-expérience}

La Figure 4 présente les résultats expérimentaux (lignes) et de simulation (pointillés) des suspensions étudiées. La Figure 4a (suspension non colloïdale de bille) illustre la sédimentation sans consolidation. En revanche, la phase de consolidation est observée pour la suspension colloïdale de kaolin (Figure 4b).
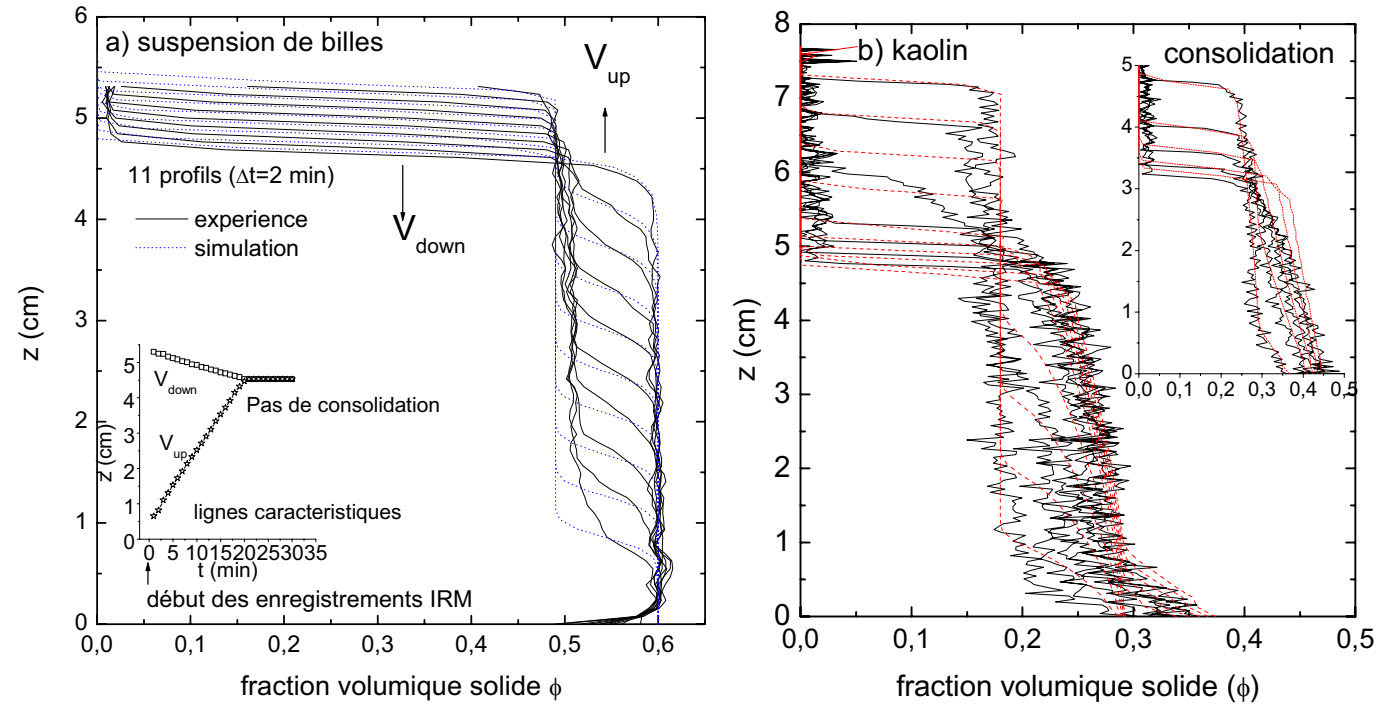

Figure 4: Comparaison expérience-simulation à partir du code 1D FE-FCT (61 nœuds) : a) sédimentation sans consolidation pour la suspension de bille (non colloïdale) illustrée par le 
diagramme des caractéristiques ; b) sédimentation et consolidation pour la suspension de kaolin (colloïdale).

En absence de consolidation (suspension non colloïdale, Figure 4a), le terme de diffusion (Eq. 4) est retiré pour la simulation. En supposant une fonction d'entravement, Eq. (10) de Leighton \& Acrivos (Acrivos et al [1]), avec la fonction de viscosité relative, Eq. (11) de Krieger-Dougherty, il ne reste que deux paramètres $\left(\phi_{\max }, n\right)$ à déterminer puisque la vitesse de Stokes et la viscosité du fluide suspendant sont connus. Pour un choix particulier de $\left(\phi_{\max }, n\right)$, l'expression de la perméabilité de Kozeny-Carman peut être retrouvée. Les résultats expérimentaux indiquent par ailleurs que $\phi_{\max }$ est égale à 0.6. Finalement, nous déterminons la valeur du paramètre $\mathrm{n}$ (égal à 1) pour obtenir un simulation 1D FEFCT en accord avec les vitesses expérimentales des ondes cinématiques, $V_{\text {up }}$ (ou $\mathrm{V}_{\text {down }}$ ) se propageant vers le haut (ou le bas resp.) et la forme des forts gradients.

$$
\begin{aligned}
& \mathrm{V}(\phi) \phi=\mathrm{K} \frac{\gamma_{\mathrm{s}}-\gamma_{\mathrm{w}}}{\gamma_{\mathrm{w}}} \phi^{2}=\mathrm{U}_{\text {stokes }} \frac{1-\phi}{\mu_{\mathrm{r}}(\phi)} \phi \\
& \mu_{\mathrm{r}}(\phi)=\frac{\mu(\phi)}{\mu_{\mathrm{f}}}=\left(1-\frac{\phi}{\phi_{\max }}\right)^{-\mathrm{n}}
\end{aligned}
$$

En présence de sédimentation et de consolidation (suspension colloïdale, Figure 4b), l'argument de Bürger et Wendland [4] est transposée à notre cas : la contrainte effective est non nulle à partir d'une valeur de la concentration $(\phi<0.28$ dans notre cas) que les auteurs relient à un concentration de percolation. Autrement dit, un régime de sédimentation sans consolidation se produit tout d'abord pour des concentration $\phi$ inférieures à 0.28 . En affectant dans l'expression de la viscosité relative, Eq. (11), la valeur 0.28 à $\phi_{\max }$, la vitesse $\mathrm{V}(\phi)$ s'annule pour $\phi=0.28$. Et l'ajustement des simulations sur les données expérimentales permet de trouver la valeur de l'exposant $n=2$. Ensuite, pour des concentrations supérieures à 0.28 , le flux de sédimentation est nul tandis que la contrainte effective cesse de l'être: en injectant une loi puissance tenant compte d'une concentration de blocage ( $\left.\phi_{\max }\right)$, l'équation suivante est obtenue :

$$
\mathrm{D}(\phi) \frac{\partial \phi}{\partial \mathrm{x}_{3}}=\frac{\mathrm{K} \phi}{\gamma_{\mathrm{w}}} \frac{\partial \sigma^{\prime}}{\partial \mathrm{x}_{3}}=\mathrm{U}_{\text {stokes }}(1-\phi) \phi\left(1-\frac{\phi}{\phi_{\mathrm{MAX}}}\right)^{-\mathrm{N}}
$$

Cette dernière formule est donc utilisée pour $\phi$ supérieur à 0.28 : une bonne correspondance simulation-expérience sur la Figure $4 \mathrm{~b}$ est obtenue pour un couple de paramètre $\left(\phi_{\mathrm{MAX}} ; \mathrm{N}\right)$ égale à $(0.65 ; 5)$.

\section{2. Simulation avec ou sans la technique FCT}

L'intérêt de la technique FCT est illustrée (Figure 5) près de la discontinuité (ou $\mathrm{du}$ fort gradient) en $\phi$ en comparant les solutions avec la technique FCT (ligne) et sans (LW, pointillé). La solution sans FCT oscille aux voisinages des forts gradients puisqu'elle est obtenue à partir d'un schéma d'intégration temporelle d'ordre deux (LW). En revanche, la solution FCT (ligne) assure des résultats non oscillants et préserve la précision du schéma d'ordre élevé lorsqu'on est hors de la zone de fort gradient. 


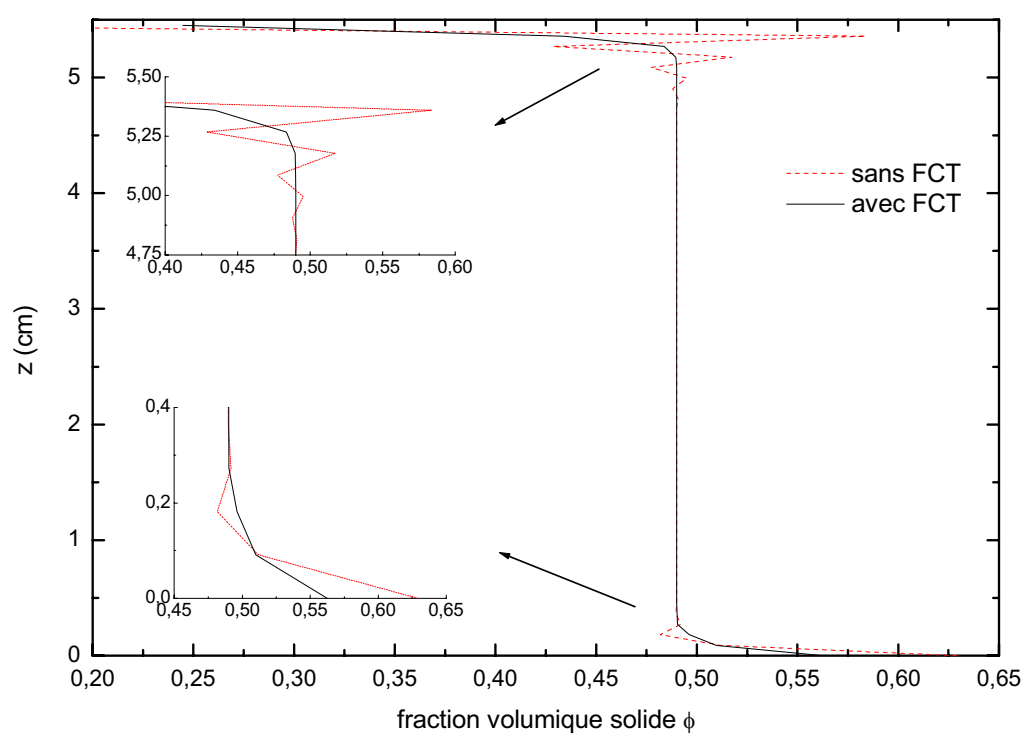

Figure 5: Comparaison au temps $\mathrm{t}=15 \mathrm{~s}$ de la solution FCT (ligne) avec la solution de Lax Wendroff (pointillé).

\section{$\underline{\text { 5. Conclusions }}$}

Un prototype IRM vertical est utilisé pour les investigations expérimentales en sédimentation-consolidation de suspension non colloïdale et colloïdale. Le phénomène de consolidation est négligeable pour le premier mais est important pour le second. La pertinence d'une technique de capture de choc telle que la technique FCT est illustrée pour un schéma d'intégration en temps d'ordre élevé (supérieur ou égal à deux).

Des développements complémentaires (et/ou supplémentaires) sont toutefois requis à la fois théoriques et expérimentaux, pour étudier la consolidation des suspensions colloïdales, et plus précisément la phase de consolidation secondaire (fluage et vieillissement) qui ne sont pas inclus dans la formulation de Gibson.

\section{$\underline{\text { Remerciements }}$}

Les auteurs remercient le LMSGC (Laboratoire des Matériaux et des Structures du Génie Civil, Institut Navier, LCPC-ENPC-CNRS UMR 113) pour la mise à disposition des données IRM sur la sédimentation et la consolidation ainsi que les conseils de Stéphane Rodts, et les discussions de l'équipe de recherche en 'Rhéophysique des pâtes et des matériaux granulaire'.

\section{Bibliographie}

1 Acrivos A., Mauri R., Fan X., "Shear induced resuspension in a couette device", Int. J. Multiphase Flow, Vol. 19, No. 5, pp. 797-802, (1993).

2 Bartholomeeusen G., Sills G.C., Znidarcic D., Van Kesteren W., Merckelbach L.M., Pyke R., Carrier W.D., Lin H., Penumadu D., Winterwerp H., Masala S., Chan D., "Sidere : numerical prediction of large-strain consolidation", Géotechnique, Vol. 52, No. 9, pp 639-648, (2002) 
3 Blanc R., Guyon E., "La physique de la sédimentation", La Recherche, Vol. 234, No. 22, pp 866-873, (1991)

4 Bürger R., Wendland W.L., "Sedimentation and suspension flows: Historical perspective and some recent developments", J. Eng.. Math., 41, pp 101-116, (2001)

5 Callaghan P.T., "Principles of Nuclear Magnetic Resonance Microscopy", Clarendon Oxford, (1991), 492p

6 Dhatt G., Touzot G., Lefrançois F., "Méthode des éléments finis", Hermès, (2005), 601p

7 Georghiou G.E., Morrow R., Metaxas A.C., "A two-dimensional, finiteelement, flux-corrected transport algorithm for the solution of gas discharge problems", J. Phys. D :Appl. Phys. 33, pp. 2453-2466, (2000)

8 Gibson R.E., Schiffman R.L., Cargill K.W., "The theory of one-dimensional consolidation of saturated clays II : finite non linear consolidation of thick homogeneous layers", Can. Geotech. J., 18, pp 280-293, (1981).

9 Raynaud J.S., Moucheront P., Baudez J.C., Bertrand F., Guilbaud J.P. and Coussot P., "Direct determination by NMR of the thixotropic and yielding behavior of suspensions", J. Rheol., Vol. 46, No. 3, pp 709-732, (2002).

10 Rodts S., Bertrand F., Jarny S., Poullain P., Moucheront P., "Développements récents dans l'application de l'IRM à la rhéologie et à la mécanique des fluides", C.R. Chimie 7, 275, (2004)

11 Toorman E.A., "Sedimentation and self-weight consolidation : general unifying theory", Géotechnique Vol. 46, No. 1, pp 103-113, (1996). 